\title{
Modeling and Simulation of a Precision Navigation System using Pseudolites Mounted on Airships
}

\author{
Burri Chandu ${ }^{1}$, Rajkumar S. Pant ${ }^{2}$ and K. Moudgalya ${ }^{3}$ \\ Indian Institute of Technology Bombay, Mumbai Maharashtra, 400076, India
}

\begin{abstract}
The availability, accuracy and integrity of the GPS signal are the three potential risks involved in using a GPS based navigation system for guidance of a moving vehicle. This paper presents the results of modeling and simulation of a system based on pseudolites (PLs) mounted on stratospheric airship platforms (SPFs), to provide precision navigation to a moving object within a specific coverage area. The specific aim of this study is to determine the effect of movements of the SPFs, and the PL monitoring time on the accuracy of positioning of a moving object. The system has been simulated in MATLAB ${ }^{\mathrm{TM}}$, and consists of a control station, six ground stations, and four PLs mounted on SPFs. The positions of the PLs on the SPFs are intermittently monitored by the ground stations, and transmitted to the control station, which calculates the exact position of the PL antenna. Using this information, the user receiver calculates its own position, which is frequently updated to provide navigation. It was seen that due to a bi-level calculation in determination of user position, the errors in determination of pseudolite position magnify the error in user positions. It was also concluded that the reduction in monitoring time substantially reduces the errors in user position determination, but will require more advanced hardware.
\end{abstract}

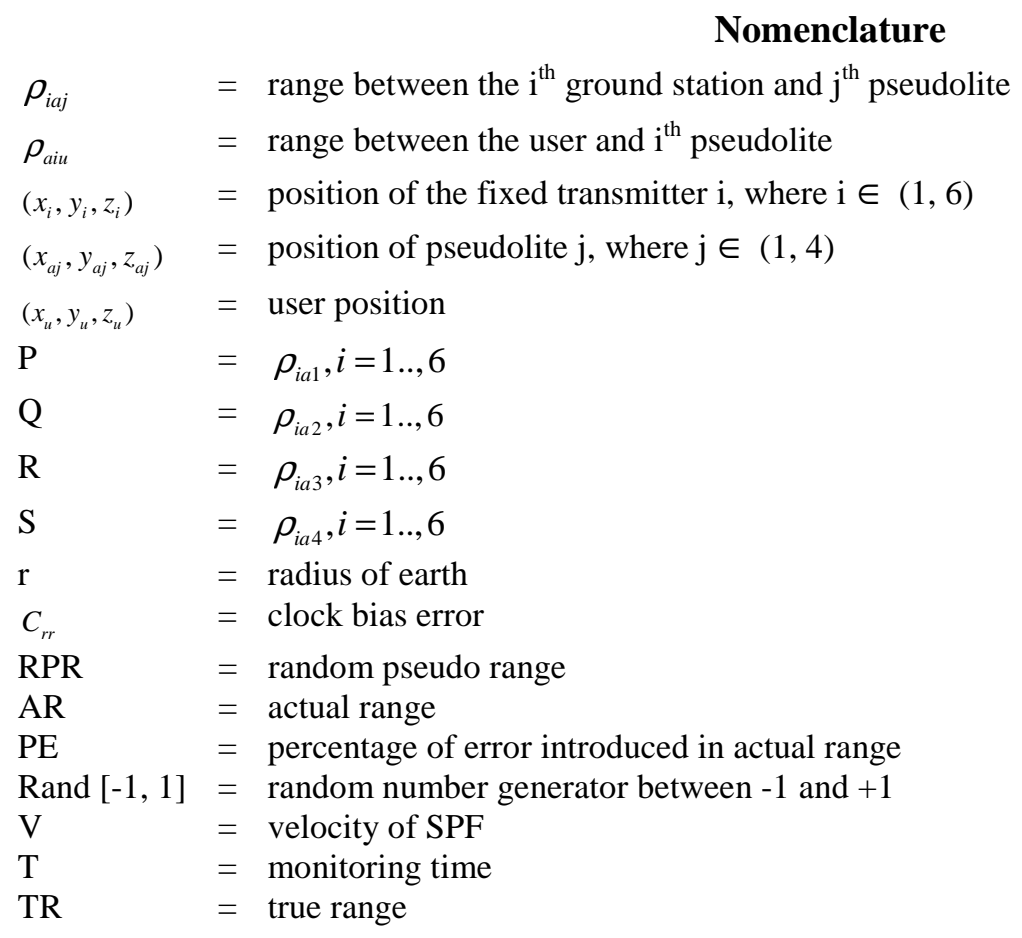

\footnotetext{
${ }^{1}$ Post Graduate Student, Aerospace Engineering Department, Powai, Non member.

${ }^{2}$ Associate Professor, Aerospace Engineering Department, Powai, Member.

${ }^{3}$ Professor, Chemical Engineering Department, Powai, Non Member.
} 


\section{Introduction}

GPS is becoming a popular system for providing navigation services, owing to its global reach and continuous availability. However, in order to maintain the integrity, availability and precision of GPS based guidance system under jamming environment, there is a need to develop a navigation system which should be able to give precise navigation solution independent of the NAVSTAR GPS satellites, at least in a local area.

One possibility of providing such a service is to mount pseudolites (pseudo satellites) on a series of high altitude platforms, and utilizing the GPS like signals emitted by them for precision navigation. The system coverage, i.e., range over which such a system can provide precision navigation, is directly dependent on the height of the platforms on which the pseudolites (PL) are mounted; higher the platform, larger the system coverage. Fixed towers have an obvious limitation for this application; both from the altitude capability, as well as the re-locatability point of view. Therefore, one has to rely on aerial platforms like fixed-wing aircraft, helicopters, or Lighter-Than-Air vehicles such as aerostats and airships.

Epley ${ }^{1}$ has shown that airships operating at stratospheric altitudes offer the best compromise among the various high altitude long endurance platforms. Such airships are proposed to be powered with an electrical propulsion system using solar regenerative fuel cells, which gives them an ability to maintain their station for very long periods of time (i.e. endurance). Their operation at stratospheric altitudes also helps in increasing their endurance, since the ambient wind speeds at such altitudes $(17-22 \mathrm{~km})$ are the least.

Tsujii et al. ${ }^{2}$ have investigated the use of a constellation of airships as stratospheric platforms (SPFs) for providing precision navigation for aerial and ground based vehicles. However, since the SPF is always moving, real-time SPF positioning and frequent broadcast of its coordinates to the user would be necessary. The precise positioning of the Pseudolite (PL) antenna on an SPF is one of the most challenging issues in providing such a service.

The basic motivation of this study is to carry out modeling and simulation of a system based on pseudolites (PLs) mounted on stratospheric airship platforms (SPFs), to provide precision navigation to a moving object within a specific coverage area. The specific aim of the study is to determine the effect of movements of the SPFs and the PL monitoring time on the accuracy of positioning of the moving object within few meters.

The proposed PL based simulation model consists of a few ground stations as well as PLs which are mounted in stratospheric platforms. The ground stations are required to continuously monitor the position of the PLs on the SPFs. By knowing the exact position of the PL antenna, the user receiver calculates its own position, which is frequently updated to provide navigation. The conceptual layout of the system is shown in Figure 1.

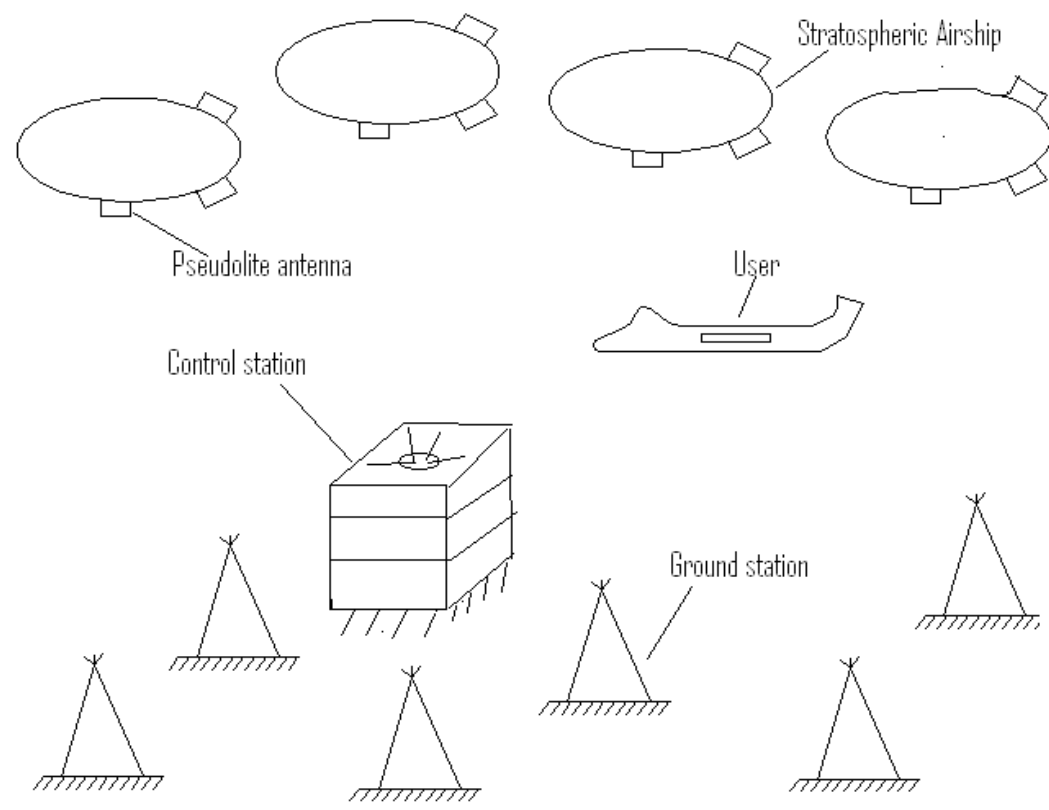

Figure 1. Conceptual layout of the precision navigation system 
The precision navigation system has been modeled in a MATLAB ${ }^{\mathrm{TM}}$ environment. The system has been decomposed into two main blocks, viz., Control Station and User Position Calculator. The Control Station block estimates the positions of the PL antennae mounted on moving SPFs, and transmits them to the PLs. The User Position Calculator uses these position updates to estimates the position of the moving object. Mathematical representation of the each block has been carried out, and validated individually. The errors in the determination of user position due to introduction of uniform and random errors in the range between the ground stations and PLs were estimated. The feasibility of the system was investigated for various symmetrical and unsymmetrical layouts of the ground stations. Finally, a sensitivity analysis of the motion of SPFs and PL monitoring time on the accuracy of the determination of the user position was carried out.

\section{Review of Literature}

\section{A. Advantages of PLs on SPF}

The advantages of PLs on SPFs compared to PLs on ground based systems have been elucidated by Tsujii et al. ${ }^{2}$ Although many applications using ground based PLs have been proposed, an operational system has not been established due to three problems specific to them, viz., 'Near-Far' problem, Multipath, and Time synchronization. These problems are present, but less severe in PLs on SPFs. The near-far problem is of concern on ground based PLs, due to smaller distances. However, these are not serious problems for PLs on a SPF. Since the height of the SPF is about $20 \mathrm{~km}$, and the distance between the PL and the user is from $20 \mathrm{~km}$ to $100 \mathrm{~km}$, the dynamic range is much less than for ground-based PL applications. The multipath of PL signals would be less because the elevation angle is rather high compared with ground PLs. Time synchronization is also not much of a problem for the PL clock on the SPF, since it can be referenced to the GPS receiver installed on the SPF, for the navigation of the SPF itself. Thus, for providing navigation over a small area, PLs mounted on SPFs are far superior to the ground based PL systems. However, the accuracy of the PL positions depends on the movement of SPFs; which can be a limiting factor for provision of precision navigation services.

\section{B. Positioning Of PL Antennae on SPFs}

There are two methods for estimating the position of PL antenna on a Stratospheric Platform, viz., GPS Transceiver method, and the Inverted GPS method. A GPS transceiver combines the function of a GPS receiver and PL. Many such devices can communicate and synchronize each other, and then estimate relative positions using the ranging information among them. If only one transceiver observes the GPS satellites, all transceivers can be referred to the precise GPS time. The Inverted GPS method is similar, but here, the GPS transceivers are replaced by the onboard PL and many ground receivers. The accuracy levels in this approach are high, and the system costs are also lower (since GPS Transceivers are very expensive). However, the disadvantage of this approach is that it requires a reference transmitter. In the present study, we use a mix of these both methods to model and simulate a system, which can fulfill our navigational aids independent of GPS in a given region.

\section{Problem Definition and Simulation Model}

We need to determine the bounds on the movement of the airship in $\mathrm{X}, \mathrm{Y}$ and $\mathrm{Z}$ directions that ensure a given accuracy in determination of the object position. This is followed by the identification of the overall model that indicates thereby identification of the individual blocks and their mathematical representations. Final simulation of all these equations is performed in MATLAB ${ }^{\mathrm{TM}}$ environment that employs algorithms of numerical techniques viz., Newton-Raphson and Least-square techniques for finding out the required coordinates. The system also includes the user position simulator model.

\section{A. Overview and working of the model}

The conceptual layout of the navigation system consists of six stations on the ground, a control station, and four PL transceivers placed in moving airships as shown in Figure 1. The frame work of the system is shown in Figure 2. 


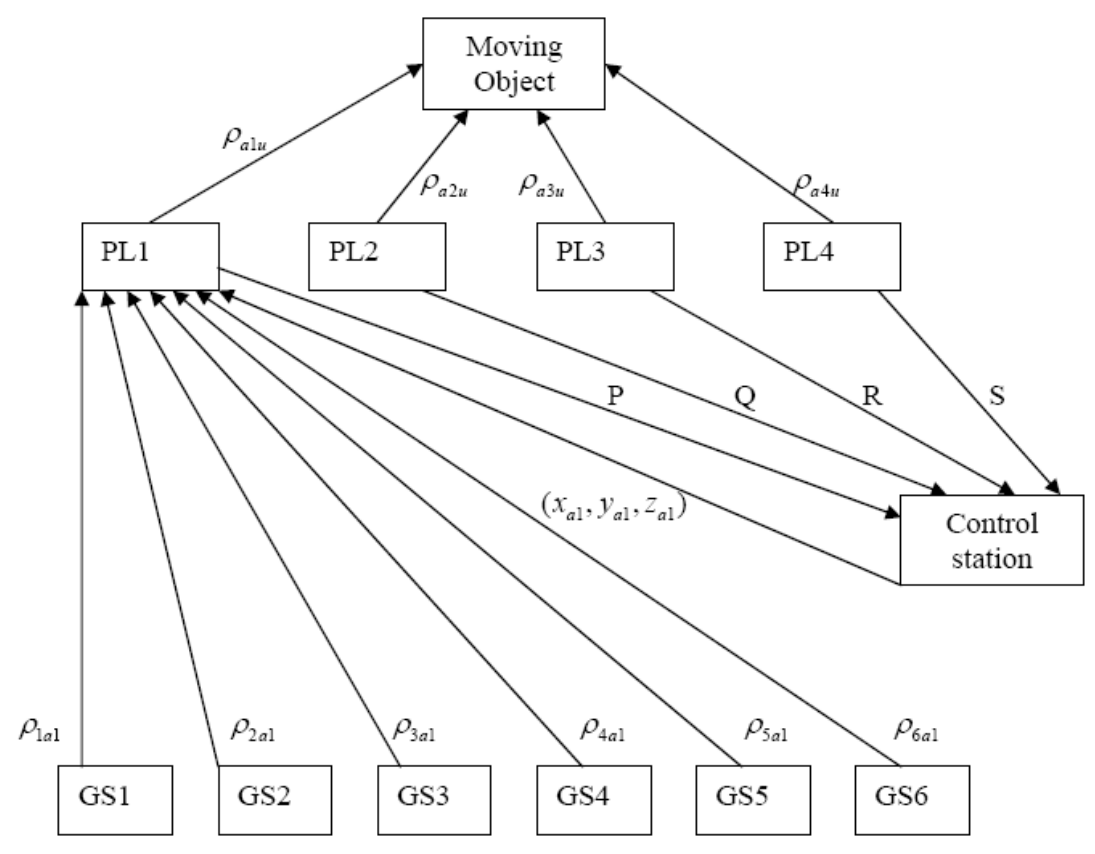

Note: For improved clarity, the lines from GS1-GS6 to PL2, PL3 and PL4 are not shown

Figure 2. Framework for the precision navigation system

Since the positions of the six ground stations are fixed, only three ground stations are required to determine the $\mathrm{X}, \mathrm{Y}$ and $\mathrm{Z}$ coordinates of the PL antenna. However, another three ground stations are required to take single difference measurement, to cancel the common errors due to factors such as tropospheric delay. These transmitters are used to estimate the exact position of the PL antenna mounted on each individual airship. This is achieved by forming six nonlinear equations for each of the PL, resulting in 24 nonlinear equations for a constellation of four PLs.

PLs consist of transceivers, which transmit signals to the Control Station, using which it (Control Station) calculates their positions (by taking the single difference measurement) and transmit it back to them. Thus, the problem is now similar to the conventional GPS system. The estimation of the position of the moving object (user position) is carried out by knowing the positions of PLs and the range vectors between them and the user.

Another framework for the model could be to delete the Control Station and install the same hardware on each of the airship platform. But this will complicate the overall system, and also increase the system cost and payload to be carried on the airships, and hence their size. Hence, these two blocks have been kept separated for simplicity.

\section{B. System Data Flow}

The sequence of data flow in the precision navigation system is as follows

1. Fixed transmitters $i=1,2, . .6$ mounted on ground stations send the ranging signals to moving PLs $j=1, \ldots .4$, mounted on SPFs.

2. Each moving PL supplies the six ranging signals to the Control Station.

3. Control Center calculates the exact position of all PLs and transmits it back to them.

4. The PLs transmit their exact position and ranging signal to the User.

5. Using the PL positions and ranging signal, the User receiver calculates its own position

In the next section, we discuss the sub blocks of the model and their mathematical representations. Effect of movement of vehicle in the time that system takes to process the mathematical equations is not considered.

\section{Mathematical representation of the model}

The system is subdivided into two individual blocks, viz., the Control Station, and User Position Calculator. 


\section{a. Control station}

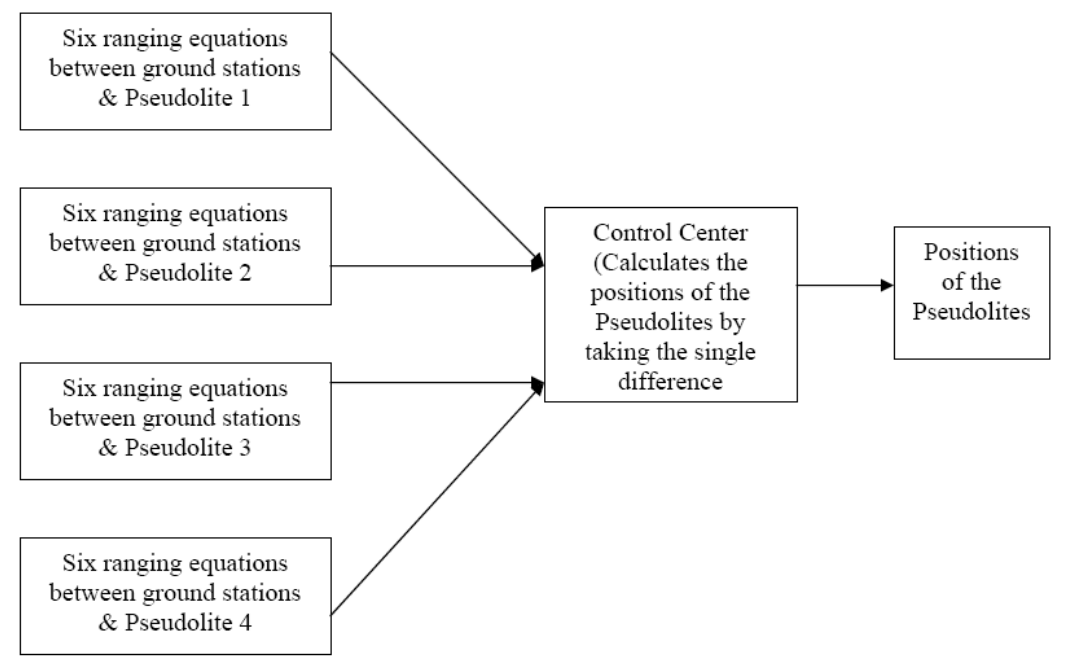

Figure 3. I/O of the Control Station

The mathematical representation of the control station is given by the set of 24 nonlinear equations representing the range vectors between the fixed transmitters and the PLs are as follows

$$
\begin{aligned}
& \left(x_{1}-x_{a 1}\right)^{2}+\left(y_{1}-y_{a 1}\right)^{2}+\left(z_{1}-z_{a 1}\right)^{2}=\rho_{1 a 1}{ }^{2} \\
& \left(x_{2}-x_{a 1}\right)^{2}+\left(y_{2}-y_{a 1}\right)^{2}+\left(z_{2}-z_{a 1}\right)^{2}=\rho_{1 a 2}{ }^{2} \\
& \left(x_{3}-x_{a 1}\right)^{2}+\left(y_{3}-y_{a 1}\right)^{2}+\left(z_{3}-z_{a 1}\right)^{2}=\rho_{1 a 3}{ }^{2} \\
& \left(x_{4}-x_{a 1}\right)^{2}+\left(y_{4}-y_{a 1}\right)^{2}+\left(z_{4}-z_{a 1}\right)^{2}=\rho_{1 a 4}{ }^{2} \\
& \left(x_{5}-x_{a 1}\right)^{2}+\left(y_{5}-y_{a 1}\right)^{2}+\left(z_{5}-z_{a 1}\right)^{2}=\rho_{1 a 5}{ }^{2} \\
& \left(x_{6}-x_{a 1}\right)^{2}+\left(y_{6}-y_{a 1}\right)^{2}+\left(z_{6}-z_{a 1}\right)^{2}=\rho_{1 a 6}{ }^{2} \\
& \text { …….... } \\
& \left(x_{6}-x_{a 4}\right)^{2}+\left(y_{6}-y_{a 4}\right)^{2}+\left(z_{6}-z_{a 4}\right)^{2}=\rho_{4 a 6}{ }^{2}
\end{aligned}
$$

The Control Station solves these equations by taking the single difference measurement. To minimize the error, the set of six non linear equations are reduced to three equations by taking the difference measurement. Thus, the resulting set of equations are exactly in the form of represented by Eqns. (1-2), (3-4) and (5-6). Solving these equations, coordinates of the first PL $\left(x_{a 1}, y_{a 1}, z_{a 1}\right)$ are generated. On the same lines, the remaining set of reduced equations can give the coordinates for rest of the PL in the form of $\left(x_{a 2}, y_{a 2}, z_{a 2}\right),\left(x_{a 3}, y_{a 3}, z_{a 3}\right)$ and $\left(x_{a 4}, y_{a 4}, z_{a 4}\right)$.

\section{b. User position calculator}

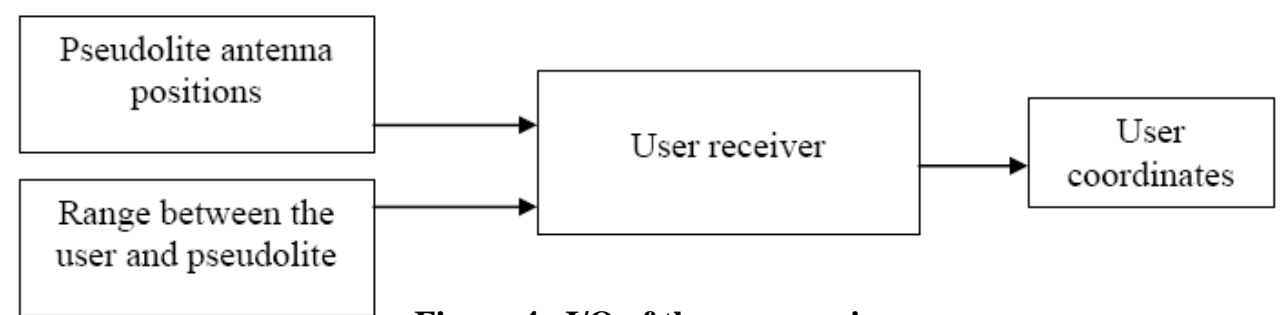

Figure 4. I/O of the user receiver 
Eqn. (25) is a nonlinear equation representing the range vectors between the first PL and the User.

$$
\begin{aligned}
& \rho_{a 1 u}{ }^{2}=\left(x_{a 1}-x_{u}\right)^{2}+\left(y_{a 1}-y_{u}\right)^{2}+\left(z_{a 1}-z_{u}\right)^{2} \\
& =x_{a 1}{ }^{2}+y_{a 1}{ }^{2}+z_{a 1}{ }^{2}+x_{u}{ }^{2}+y_{u}{ }^{2}+z_{u}{ }^{2}-2 x_{a 1} x_{u}-2 y_{a 1} y_{u}-2 z_{a 1} z_{u}
\end{aligned}
$$

By substituting $x_{u}{ }^{2}+y_{u}{ }^{2}+z_{u}{ }^{2}=r^{2}$, where $r=$ radius of earth, and introducing the clock bias error $C_{r r}$

$$
\rho_{a 1 u}{ }^{2}-\left(x_{a 1}{ }^{2}+y_{a 1}{ }^{2}+z_{a 1}{ }^{2}\right)-r^{2}=C r r-2 x_{a 1} x_{u}-2 y_{a 1} y_{u}-2 z_{a 1} z_{u}
$$

Eqns. (26-28) are the similar equations for the remaining three PLs

$$
\begin{aligned}
& \rho_{a 2 u}{ }^{2}-\left(x_{a 2}{ }^{2}+y_{a 2}{ }^{2}+z_{a 2}{ }^{2}\right)-r^{2}=C r r-2 x_{a 2} x_{u}-2 y_{a 2} y_{u}-2 z_{a 2} z_{u} \\
& \rho_{a 3 u}{ }^{2}-\left(x_{a 3}{ }^{2}+y_{a 3}{ }^{2}+z_{a 3}{ }^{2}\right)-r^{2}=C r r-2 x_{a 3} x_{u}-2 y_{a 3} y_{u}-2 z_{a 3} z_{u} \\
& \rho_{a 4 u}{ }^{2}-\left(x_{a 4}{ }^{2}+y_{a 4}{ }^{2}+z_{a 4}{ }^{2}\right)-r^{2}=C r r-2 x_{a 4} x_{u}-2 y_{a 4} y_{u}-2 z_{a 4} z_{u}
\end{aligned}
$$

The above four linear equations can be represented in the Matrix form as shown in Eqn. (29)

$$
\left(\begin{array}{l}
\rho_{a 1 u}{ }^{2}-\left(x_{a 1}{ }^{2}+y_{a 1}{ }^{2}+z_{a 1}{ }^{2}\right)-r^{2} \\
\rho_{a 2 u}{ }^{2}-\left(x_{a 2}{ }^{2}+y_{a 2}{ }^{2}+z_{a 2}{ }^{2}\right)-r^{2} \\
\rho_{a 3 u}{ }^{2}-\left(x_{a 3}{ }^{2}+y_{a 3}{ }^{2}+z_{a 3}{ }^{2}\right)-r^{2} \\
\rho_{a 4 u}{ }^{2}-\left(x_{a 4}{ }^{2}+y_{a 4}{ }^{2}+z_{a 4}{ }^{2}\right)-r^{2}
\end{array}\right)=\left(\begin{array}{llll}
-2 x_{a 1} & -2 y_{a 1} & -2 z_{a 1} & 1 \\
-2 x_{a 2} & -2 y_{a 2} & -2 z_{a 2} & 1 \\
-2 x_{a 3} & -2 y_{a 3} & -2 z_{a 3} & 1 \\
-2 x_{a 4} & -2 y_{a 4} & -2 z_{a 4} & 1
\end{array}\right)\left(\begin{array}{c}
x_{u} \\
y_{u} \\
z_{u} \\
C_{r r}
\end{array}\right)
$$

Unknown state vector $=\left(\begin{array}{llll}x_{u} & y_{u} & z_{u} & C_{r r}\end{array}\right)^{T}$

$R=M U_{p}$

Where $R=$ vector (known), $M=$ matrix (known) and $U_{p}=$ vector (unknown)

$$
\begin{aligned}
M^{-1} R & =M^{-1} M U_{p} \\
& =U_{p} \\
& =\left(\begin{array}{llll}
x_{u} & y_{u} & z_{u} & C_{r r}
\end{array}\right)^{T}
\end{aligned}
$$

The overall system has been implemented in MATLAB ${ }^{\mathrm{TM}}$. The next section provides description of the various user defined functions in the code.

\section{Explanation of code}

The code consisting of four user defined functions. They are PLC, F4PLC, UPC and F4UPC. PLC function takes input as the positions of the ground stations and the range between ground stations and PL antenna and estimate the exact position of the PL antenna. F4PLC is the subordinate function of the above function. An initial guess need to be supplied for the PL position in this function. The function progresses towards the converged solution from the given initial guess (Uses least square algorithm to calculate the position of PLs). This function helps in solving the position of the PLs. UPC takes input as the calculated PL positions from PLC function and the range between the PL antenna and user. This function finally gives the exact position of user by taking the above input. F4UPC is the subordinate function of the above function. An initial guess need to be supplied for user position in this in this function. The function progresses towards converged solution of user from the given initial guess. (This also uses least square technique to calculate the exact position of user coordinates).

The model described above was tested to verify its robustness and convergence for various patterns of the ground stations. Sensitivity analysis of the variation of error in calculating the user position with monitoring time and movement of PL in $\mathrm{X}, \mathrm{Y}$ and $\mathrm{Z}$ has been carried out. The results of these investigations are presented in the next section.

\section{Results and Discussion}

\section{A. Verification of the code}

a) The solver was seen to converge to the same final point even with different initial guess, this shows that the code is independent of initial guess. In one such example, it was seen that in both the cases, the code converges to the same point viz. [-600m, 2300m, $-350 \mathrm{~m}]$ even when we start from $[300 \mathrm{~m}, 300 \mathrm{~m}, 300 \mathrm{~m}]$ as shown in Figure 5 or $[-300 \mathrm{~m},-300 \mathrm{~m},-300 \mathrm{~m}]$ as shown in Figure 6. 


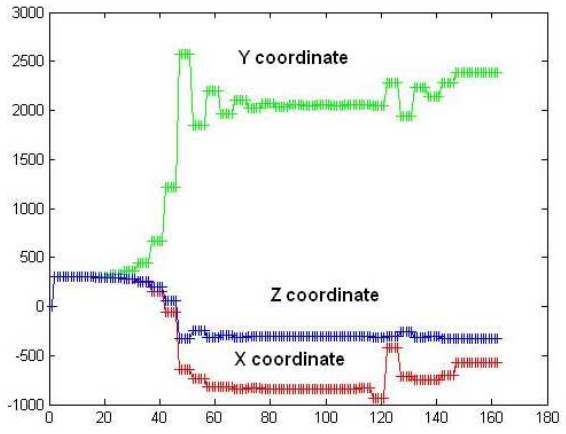

Figure 5. Trajectory of the user coordinates with initial guess as $[300 \mathrm{~m}, 300 \mathrm{~m}, 300 \mathrm{~m}]$

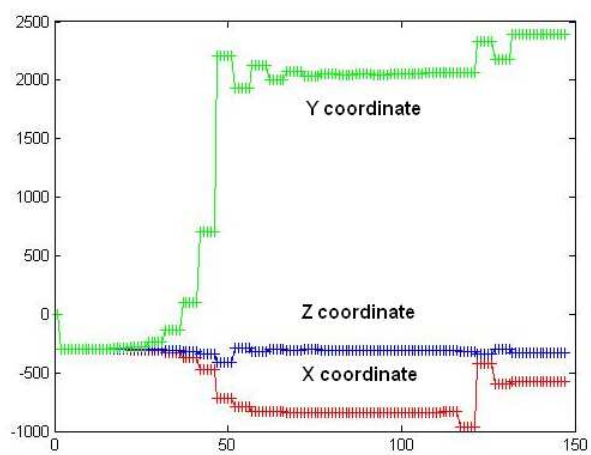

Figure 6. Trajectory of the user coordinates

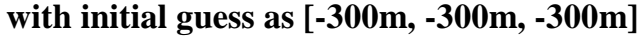

b) The solver solution converged in two iterations when the solution itself was supplied as the initial guess.

c) The PL positions were assumed and the ranges between the PLs calculated manually. When the calculated ranges were supplied to the model, it resulted in the assumed PL positions, as shown in Table 1 for some trial runs.

\begin{tabular}{|c|c|c|c|c|c|c|}
\hline \multirow{2}{*}{$\begin{array}{l}\text { Pseudolite No } \\
1\end{array}$} & \multicolumn{3}{|c|}{ Assumed positions of the pseudolites (m) } & \multicolumn{3}{|c|}{ Positions with inverted calculation (m) } \\
\hline & 120 & 80 & 40 & 120.32 & 78.08 & 40.59 \\
\hline 2 & 200 & 100 & 50 & 200.03 & 99.79 & 50.06 \\
\hline 3 & 150 & 100 & 30 & 149.78 & 99.75 & 30.21 \\
\hline 4 & 100 & 150 & 20 & 99.98 & 150.11 & 19.96 \\
\hline
\end{tabular}

Table 1. Deviation of the pseudolite positions from assumed to calculated

These tests established the robustness of the code. In the next section, the results of feasibility of various configurations of the ground stations are presented.

\section{B. Comparison of feasibility of various configurations}




\begin{tabular}{|l|l|l|l|}
\hline \multicolumn{1}{|c|}{ Configuration } & $\begin{array}{l}\text { Initial } \\
\text { guess } \\
\text { dependence }\end{array}$ & Feasibility & Remarks \\
\hline Circle configuration at constant height & YES & Not feasible & \\
\hline $\begin{array}{l}\text { Circle configuration with an inclination of } 30 \\
\text { degree about X-axis }\end{array}$ & YES & Not feasible & \\
\hline $\begin{array}{l}\text { Circle configuration with an inclination of } 30 \\
\text { degree about X and Y-axis }\end{array}$ & YES & Not Feasible & \\
\hline Hexagon configuration at constant height & NA & Not feasible & No solution obtained \\
\hline $\begin{array}{l}\text { Hexagon configuration with an inclination of 30 } \\
\text { degree about X-axis }\end{array}$ & YES & Not feasible & \\
\hline $\begin{array}{l}\text { Hexagon configuration with an inclination of } 30 \\
\text { degree about X and Y-axis }\end{array}$ & NO & Feasible & $\begin{array}{l}\text { PL should not be at the } \\
\text { centre of configuration }\end{array}$ \\
\hline $\begin{array}{l}\text { Triangular configuration at constant height } \\
\text { Triangular configuration with an inclination of 30 } \\
\text { degree about X-axis }\end{array}$ & NO & Feasible & $\begin{array}{l}\text { No solution } \\
\text { obtained }\end{array}$ \\
\hline $\begin{array}{l}\text { Triangular configuration with an inclination of } 30 \\
\text { degree about X and Y-axis }\end{array}$ & NO & Feasible & $\begin{array}{l}\text { PL should not be at the } \\
\text { centre of configuration }\end{array}$ \\
\hline
\end{tabular}

Table 2. Comparison of the various configurations of ground stations

\section{Testing the algorithm for random arrangement of Ground Stations}

Compared to symmetric configuration, much better results have been observed with the random configuration of the ground stations. For the random configuration of ground stations, the solution was found to be completely independent of initial guesses. Further, the solver converged even if the range between the PL and all ground stations were equal. Therefore, it was observed that random arrangement of ground stations is a much better option. Table 3 shows the positions of ground stations and PLs.

\begin{tabular}{|l|l|l|l|}
\hline & $\mathbf{X}(\mathbf{m})$ & $\mathbf{Y}(\mathbf{m})$ & $\mathbf{Z}(\mathbf{m})$ \\
\hline Ground station positions & $200( \pm) 100$ & $19000( \pm) 1000$ & $300( \pm) 100$ \\
\hline Pseudolite positions & $5000( \pm) 500$ & $18500( \pm) 1000$ & $15000( \pm) 1000$ \\
\hline
\end{tabular}

Table 3. Location of the ground stations and pseudolite positions

The next section presents the result of effect of error in determination of range between PL and ground station on the determination of PL coordinates and user coordinates.

\section{Sensitivity analysis}

Sensitivity analysis was carried out for the random arrangement of ground stations. Details of the same are discussed in the section that follows.

\section{a. The effect of uniform range error in the calculation of the PL position}

It is important to determine the effect of PL positions, when there is an error in the measured range. Figure 10 shows the effect of "Percentage uniform error" in the range between PL and ground stations on the "Percentage error in the positions" of the PL for $\mathrm{X}, \mathrm{Y}, \mathrm{Z}$ coordinates. Both positive and negative errors in the range of the PL were considered. It was observed that as the uniform error in range increases, the error in the position of the PL was also increases in a linear fashion, both in positive and negative direction. The error in Y coordinate, (both due to positive and negative error) was seen to be insignificant. This is because the PL and ground stations were not allowed a large variation in the $\mathrm{Y}$ coordinate. 


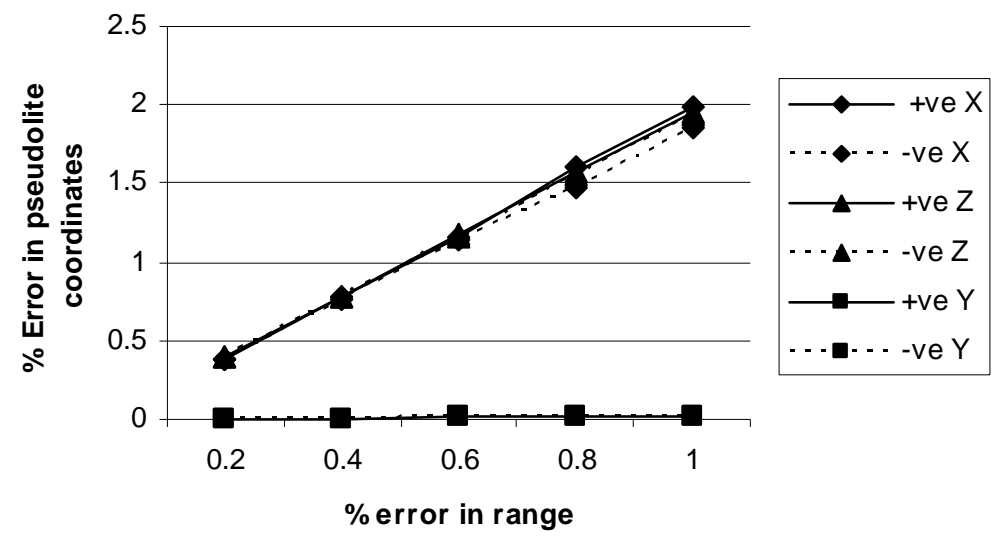

Figure 10. Percentage error in pseudolite co-ordinates v/s percentage error in the range of pseudolite

\section{b. The effect of uniform range error in the calculation of the user position}

From Figure 10 it can be concluded that a nonlinear relation exists between the range error and user position error. Due to bi-level calculation the error in the PL positions magnify the errors in the user position.

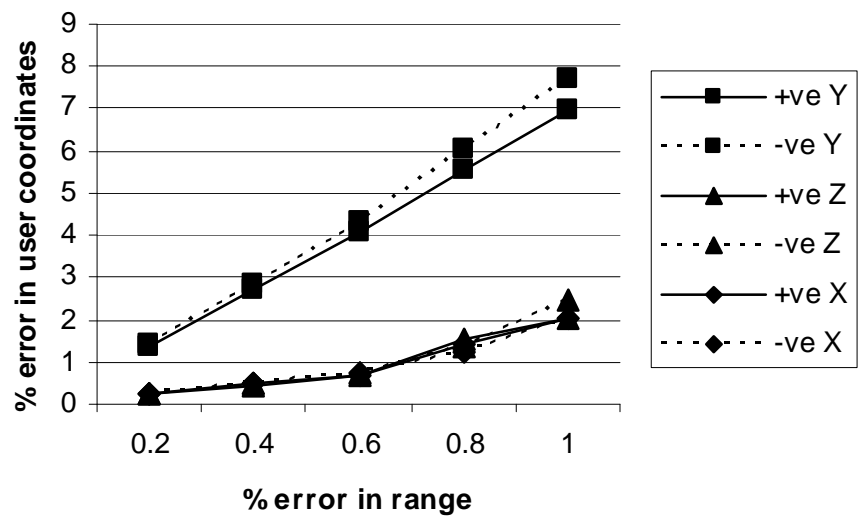

Figure 11. Percentage error in the user coordinates v/s percentage error in the range of pseudolite

\section{c. Effect of random error in the range on PL and user coordinates}

Next, the effect of random error on user coordinates was examined. The random pseudo range can be formulated as shown in Eqn. (33).

$\mathrm{RPR}=\mathrm{AR}+\mathrm{Rand}[-1,1] * \mathrm{PE}$

Figure 12 shows percentage error in PL coordinates with the variation of the percentage random error in the range.

From Figure 12 it is clear that as we increase the random error, the error in the PL co-ordinates increases linearly. A comparison with Figures 4 and 6 shows that the error in PL coordinates due to random error is nearly double of the error in coordinates due to uniform error. 


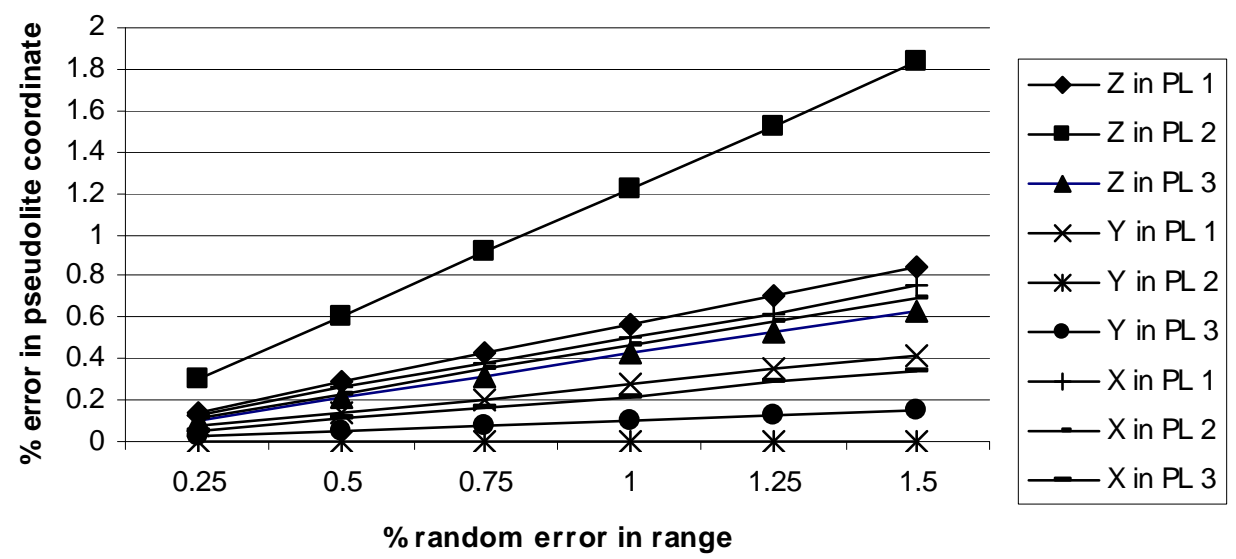

Figure 12. Percentage error in pseudolite co-ordinates v/s percentage of random error in range

\section{d. Effect of random error in the range on user coordinates}

Figure 13 shows percentage error in user coordinates with the variation of the percentage of random error in the range.

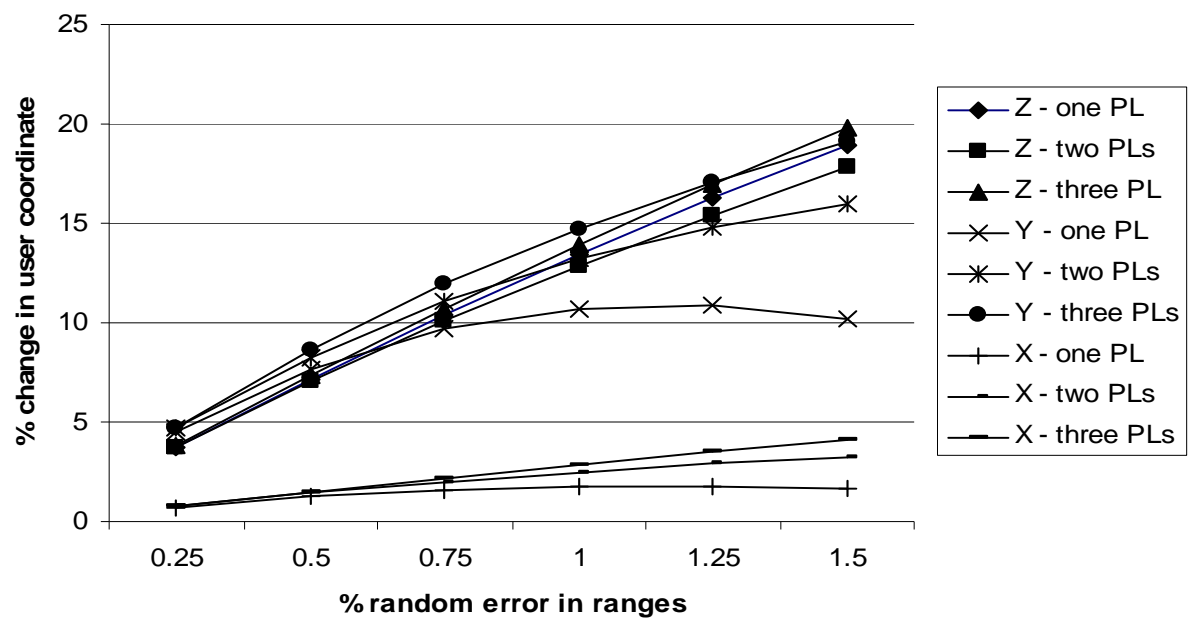

Figure 13. Percentage Change in User coordinate v/s Percentage random error in range

From Figure 13 it is clear that as the random error in range is increased the error in $\mathrm{Z}$ coordinates also increases and they are more in magnitude when three or two PLs are moving. In case of the Y coordinate, however the error reaches a peak and then tends to decrease. Further, the errors in $\mathrm{X}$ coordinate are the least in all cases. A comparison with Fig 4.7 shows that the error in user coordinates due to random error is nearly double of the error in coordinates due to uniform error.

\section{E. Effect of PL monitoring time and movement on the accuracy of user position}

The two parameters which can affect the accuracy of the system considerably are the PL monitoring time and the movement of the PL. The monitoring time is defined as the minimum time required for monitoring the PL. There must be a tradeoff between these two parameters. It is clear that when the monitoring time is large, the accuracy is poor. i.e., the accuracy is inversely proportional to monitoring time. The accuracy also reduces if the movement in 
the SPF is large. The coordinates deviate from their original position due to the random movement in the PL positions, which can be obtained from Eqn. (34).

Random displacement of SPF $=\left(\mathrm{V}^{*} \mathrm{~T} * \mathrm{R}\right.$ and $\left.[-1,1]\right)$

The range between the SPF and fixed receiver will change because of the random movement in the SPF. The true range TR can be obtained from Eqn. (35) as

$\mathrm{TR}=$ Range between the SPF and fixed transmitter $+(\mathrm{V} * \mathrm{~T} * \mathrm{Rand}[-1,1])$

Figure 14 shows the variation of user coordinates with the variation of monitoring time for fixed movement of SPF. It is clear that as the monitoring time increasing for the given movement of SPF, the accuracy levels are decreasing.

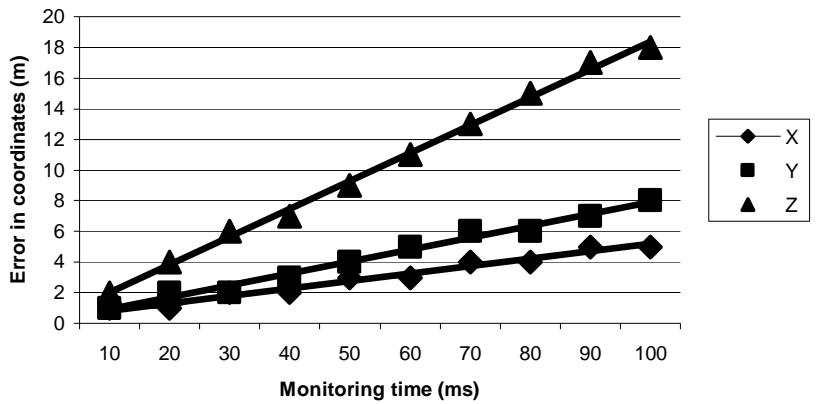

Figure 14. Error in the coordinates for a fixed movement in SPF v/s monitoring time

The movement in the SPF is an independent parameter, which cannot be changed and it completely depends on the dynamics of the SPF and the ambient atmospheric conditions. There are no exact models readily available which can model the dynamics of the SPF. The quantity which can be easily varied is the monitoring time of the PL. The hardware puts the constraints on the monitoring time. Figure 15-17 show the variation of error in user coordinates with the variation of PL movement from $0.1 \mathrm{~m} / \mathrm{sec}$ to $1 \mathrm{~m} / \mathrm{sec}$. The graphs are plotted for various monitoring time values.

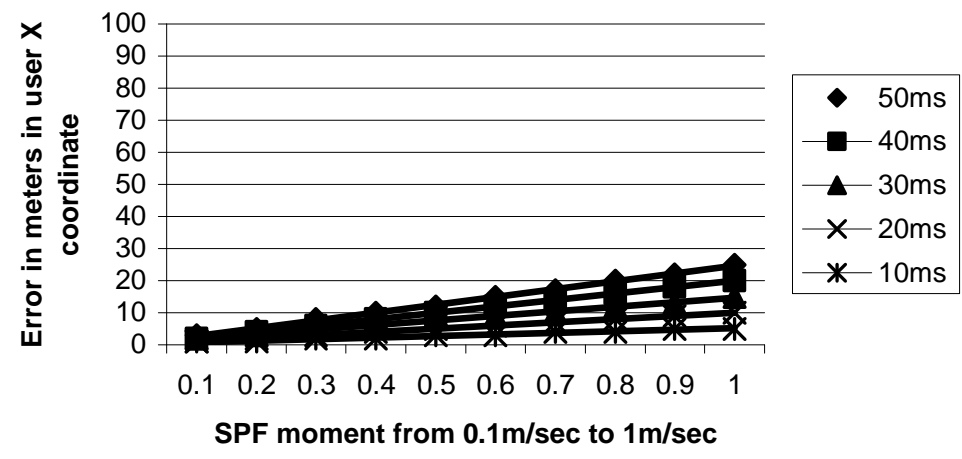

Figure 15. Error in User coordinate $X$ for various monitoring times v/s SPF velocity 


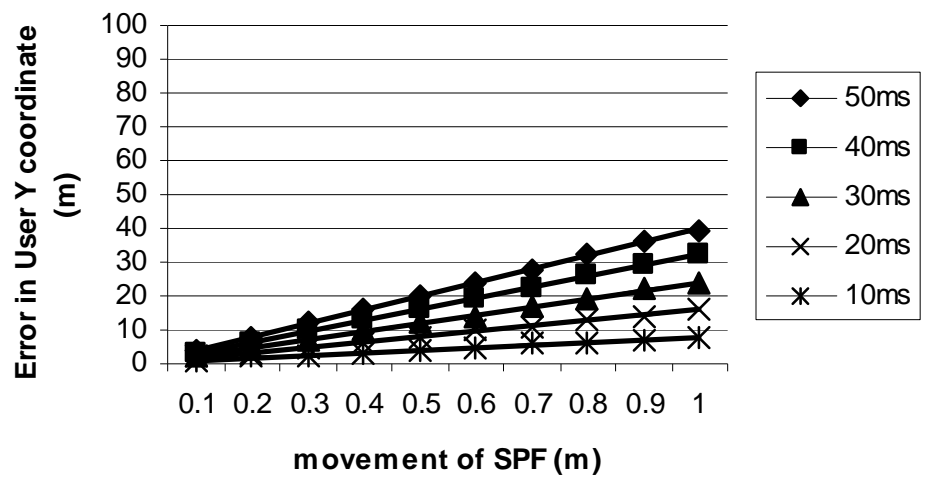

Figure 16. Error in User coordinate $Y$ for various monitoring times v/s SPF velocity

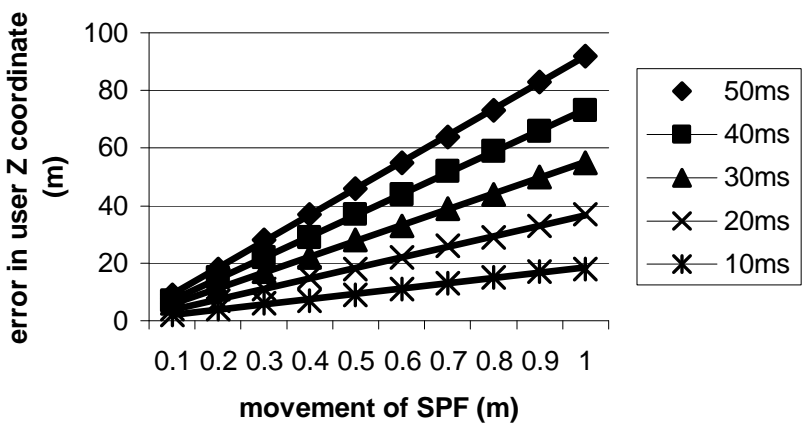

Figure 17. Error in User coordinate $Z$ for various monitoring times v/s SPF velocity

From Fig 15-17, it is clear that as the movement of the SPF increases, the error in the coordinates is higher for a given monitoring time. In these Figures, the lines shift up wards (error level increases) as we increase the monitoring time. So the accuracy is inversely proportional to monitoring time and the movement of the SPF. These Figures help us in carrying out a tradeoff analysis between the monitoring time and the movement of SPF. It is always a challenge for the designers to select these design parameters for a given level of accuracy.

The next section lists the conclusions of the study and provides pointers for the future work to be carried out.

\section{Conclusions and future work}

\section{A. Conclusions}

In this study, the modeling and simulation of precision navigation system using PLs mounted on four stratospheric airships and six ground stations along with a control station has been carried out. This system helps in determining the position of a moving aerial vehicle without depending on GPS signals.

The key conclusions from this study can be summarized as follows;

i. The ground stations should not be kept in a symmetric configuration. Such configuration results in infeasible solutions, especially when the PL ranges are equal from the ground stations.

ii. In the present study, the errors in PL Y coordinate due to the error in the range between the PL and ground station coordinate are very less. This is only because both PL and ground stations were not allowed a large variation in the $\mathrm{Y}$ coordinate, and not a general trend.

iii. There is a bi-level calculation in determination of user position. Hence, the errors in determination of PL position magnify the error in user position. 
iv. The error in coordinates due to random error is nearly double of the error in coordinates due to uniform error in the ranges. Hence it can be concluded that the determination of user position is more effected by random errors (such as tropospheric \& atmospheric) as compared to uniform error (such as clock bias).

v. It is essential to restrict and accurately estimate the movement of SPFs, since the errors due to these movements are large.

vi. The reduction in monitoring time substantially reduces the errors, but will require more advanced hardware.

\section{B. Future work}

In the present work, the effect of size of the area in which the SPFs operate has not been considered. The size of the area of operation affects the GDOP (Geometric Dilution of Precision). The motion of the PLs can be more accurately predicted by inserting a six degree of freedom dynamics model, and then the results will be more accurate and useful. We can model the various errors corresponding to PLs by the practical experiments, by which we can improve the accuracy of the model. The effect of loss of PLs on the user accuracy can also be investigated.

\section{References}

${ }^{1}$ Epley, L. E., "Stratospheric Aircraft, Blimps, Balloons and Long Endurance Vehicles", Future Aeronautical and Space Systems, Eds. Noor, A. K. and Venneri, S. L., Progress in Astronautics and Aeronautics, Vol. 172, American Institute of Aeronautics and Astronautics, 1997.

${ }^{2}$ Tsujii, T., Rizos, C., Wang, J., Dai, L. and Roberts, C., “A Navigation/Positioning Service Based on Pseudolites Installed on Stratospheric Airships", $5^{\text {th }}$ International Symposium on Satellite Navigation Technology \& Applications, Australia, 2001.

${ }^{3}$ Liwen, D., Wang, J., Tsujii, T. and Rizos, C., "Pseudolite applications in positioning and navigation: Modeling and geometric analysis", The University of New South Wales, Australia, November, 1998.

${ }^{4}$ Wang, J., Tsujii, T. and Rizos, C., "Estimation of Residual Tropospheric Delay for High-Altitude Vehicles: Towards Precise Positioning of a Stratosphere Airship", School of Geomatic Engineering, University of New South Wales, Australia, 2000.

${ }^{5}$ Wang, J., Tsujii, T., Rizos, C. and Liwen, D., "A Technique for Precise Positioning of High Altitude Platforms System (HAPS) Using a GPS Ground Reference Network", School of Surveying and Spatial Information Systems, The University of New South Wales, Australia, 2001.

${ }^{6}$ Tsujii, T. and Harigae, M., "Experiments of Inverted Pseudolite Positioning For Airship-Based GPS Augmentation System”, Flight Systems Research Center, National Aerospace Laboratory, Japan, 2002.

${ }^{7}$ Liwen, D., Wang, J., Tsujii, T. and Rizos, C., "Inverted Pseudolite Positioning and its Applications", The University of New South Wales, Australia, April 2001.

${ }^{8}$ Stewart, H. C., "GPS Pseudolites: Theory, Design and Applications", Stanford University Press, USA, September, 1997.

${ }^{9}$ Wang, J., Rizos, C. and Joel, B., "The Performance of a Pseudolite-Based Positioning System for Deformation Monitoring", School of Surveying and Spatial Information Systems, Australia, 2002.

${ }^{10} J o n a t h a n$, S. M., Edward, L. A., David, P. J., and Stephen, R., "GPS Pseudolite Transceivers and their Applications", Stanford University, USA, 1999.

${ }^{11}$ Tsujii, T., Harigae, M., Barnes, J., Wang, J. and Rizos, C., "Experiments of Inverted Pseudolite Positioning for Airship-Based GPS Augmentation System", $6^{\text {th }}$ International Symposium. on Satellite Navigation Technology \& Applications, 2002.

${ }^{12}$ Grewal, M. S., Global positioning systems, inertial navigation and integration, 3rd ed., John Wiley and Sons, 2003, Chaps. 1, 2.

${ }^{13}$ Schilling, R. J., Applied Numerical Methods for Engineers using MATLAB and C, 1st ed., Thomson Books, 2000, Chap. 5, pp. 228-232. 